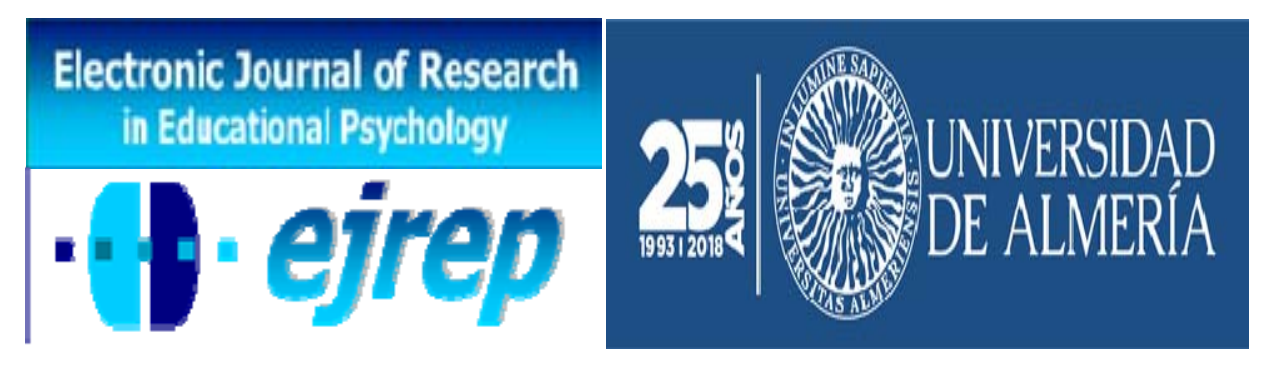

Tlustre Colegio Oficial

L DE AMOALUCÍA ORIENTA.

\title{
Práctica de actividad física, conducta prosocial y autoconcepto en adolescentes: conexiones en el contexto escolar
}

\author{
Francisco Daniel Martínez-Martínez ${ }^{1}$ y Juan González- \\ Hernández ${ }^{2}$ \\ ${ }^{1}$ Consejería de Educación de la Región de Murcia, Murcia \\ ${ }^{2}$ Dpto. Personalidad, Evaluación y Tratamiento Psicológico, Universidad de \\ Granada, Granada
}

\section{España}

Correspondencia: Francisco Daniel Martínez Martínez. CEIP San Antonio. Calle Juanmi de Jumi, 5, 30876, Cañada de Gallego (Mazarrón). España. E-mail: fdaniel.martinez@um.es

(C) Universidad de Almría and Ilustre Colegio Oficial de la Psicología de Andalucía Oriental (Spain) 


\section{Resumen}

Introducción. La investigación tiene dos objetivos: (1) establecer diferencias entre variables internas (autoconcepto y prosocialidad) y externas (frecuencia de actividad física) que rodean al adolescente en el contexto escolar, según condiciones sociales (sexo, tipo de práctica de actividad física y edad. (2) Proponer relaciones de causalidad y predictibilidad entre las diferentes dimensiones del autoconcepto y conducta prosocial hacia la práctica de actividad física en una muestra de adolescentes.

Método. Se trata de un estudio de carácter descriptivo y de asociación lineal, cuya muestra está formada por 409 participantes de ESO y Bachillerato. Se ha utilizado un cuestionario sociodemográfico, el Cuestionario de Autoconcepto (AF-5), la Escala de prosocialidad en adolescentes y adultos, y la Escala de Dificultades de Socialización de Cantoblanco (SOC).

Resultados. El principal ámbito donde se fomentan las relaciones sociales en la adolescencia es en el de los amigos (42.3\%), seguido de la escuela (34\%), señalando el deporte como el entorno menos propicio para fomentar sus relaciones sociales (42.1\%). La práctica de actividad física, se relaciona positivamente con las dimensiones de autoconcepto, excepto con el emocional, contemplando una relevante influencia de la condición de estar federado, para tal relación negativa. Además, se confirma la relación entre autoconcepto y conducta prosocial para una respuesta adaptada psicosocialmente, de cara al aumento de la frecuencia de práctica de actividad física en la adolescencia.

Discusión y Conclusiones. La práctica de actividad física provoca que los adolescentes tengan una mejor percepción de sus propias condiciones personales, aumentando así su confianza para establecer relaciones sociales con compañeros y docentes, en el desarrollo de actitudes de búsqueda de experiencias sociales positivas. Todo ello contribuyendo al aumento de la frecuencia de la práctica deportiva, mediada por el entendimiento y comprensión de su bienestar ante las conductas prosociales.

Palabras claves: diferencias individuales, prosocialidad, educación física, jóvenes, escuela. 


\section{Abstract}

Introduction. The research has two objetives: to establish differences between internal variables (self-concept and pro-sociality) and external variables (frequency of physical activity) that surround adolescents in the school context, according to social conditions (sex, type of physical activity and age); and to propose causality and predictability relationships between the different dimensions of self-concept and prosocial behavior towards the practice of physical activity in a sample of adolescents.

Method. This is a descriptive and lineal association study, whose sample is made up of 409 participants of ESO and Bachillerato. A sociodemographic questionnaire has been used, the self-concept questionnaire (AF-5), the scale of pro-sociality in adolescents and adults, and the Cantoblanco scale of socialization difficulties (SOC).

Results. The main area where social relations are encouraged in adolescence is friends (42.3\%), followed by school (34\%), indicating sport as the least favorable environment to foster their social relations (42.1\%). The practice of physical activity is positively related to the dimensions of self-concept, except for the emotional, contemplating a relevant influence of the condition of being federated, for such a negative relationship. In addition, the relationship between self-concept and prosocial behaviour is confirmed for a psychosocially adapted response to the increased frequency of physical activity in adolescence.

Discussion and Conclusion. The practice of physical activity causes adolescents to have a better perception of their own personal conditions, thus increasing their confidence to establish social relationships with peers and teachers, in the development of attitudes seeking positive social experiences. All this contributes to the increase in the frequency of sports practice, mediated by the understanding and comprehension of their well-being in relation to prosocial behaviors.

Key words: individual differences, pro-sociality, physical education, youth, school. 


\section{Introducción}

El desarrollo que se genera en la adolescencia a nivel madurativo, personológico y cognitivo, juega un papel extremadamente relevante en las relaciones sociales y el entendimiento individual de las mismas (Rowsell, Ciarrochi, Deane, y Heaven, 2016). Los adolescentes dejan de tener como principal referencia a sus padres y pasan más tiempo fuera de casa, lo que conduce a un menor número de interacciones positivas con sus progenitores, apareciendo conflictos relacionados con aspectos de la vida cotidiana tales como las tareas del hogar, nuevas amistades, formas de vestir o la hora de volver a casa (Darr, 2011; Wang y Holcombe, 2010), abandono del deporte (Janosz, Archambault, Morizot y Pagan, 2008; Li, Doyle, Kalvin, Liu y Lerner, 2011), y rendimiento escolar (Martínez, Inglés, Piqueras y Ramos, 2010). Por otro lado, aumenta la importancia, intensidad y estabilidad de las relaciones con los amigos, y el grupo de iguales se convierte en el contexto de socialización más influyente (Martínez y González, 2017; Vanatta, Gartsein, Zeller y Noll, 2009).

La familia se considera una institución fundamental en el desarrollo del individuo y su socialización (Fajardo, Maestre, Felipe, León y Polo, 2017). De hecho, autores como Oyarzún, Estrada, Pino y Oyarzún (2012), han concluido que el apoyo familiar, la aceptación de uno mismo y la amistad o las buenas relaciones sociales que se puedan dar en el entorno escolar, durante las primeras etapas socializadoras, son algunos de los factores que tienen relación directa con el autoconcepto, el desarrollo cognitivo y la práctica de actividad física.

En este sentido, elementos relacionados con las diferencias individuales (empatía, conducta prosocial o autoconcepto), permiten comprender la variabilidad de los comportamientos en el adolescente, así como la influencia sobre la respuesta psicológica que le permita ajustarse psicosocialmente (Caprara y Cervone, 2000; Martínez y González, 2017). Precisamente, un buen indicador psicológico del ajuste escolar es el autoconcepto (Wang y Fredricks, 2014), definido como: "conocimiento o representación cognitiva que cada individuo posee con relación a sus características personales, atributos y limitaciones, se corresponde con la imagen que cada uno tiene de sí mismo, sin la necesidad de someterse a comparación con otras personas" (De la Torre, García, Carpio y Casanova, 2008, p. 58).

Durante años entendido como constructo unidimensional, se sostiene que los individuos generan percepciones de sí mismos muy diferentes en distintos aspectos de sus vidas 
(familiar, social, emocional, físico...), considerando más apropiado estudiar por separado cada una de sus dimensiones (Goñi e Infante, 2010; Rosenberg, 1979; Stojiljković, Todorović, Đigić y Dosković, 2014).

Por otra parte, la conducta prosocial se ha definido como un conjunto de acciones que se realizan de forma voluntaria para aumentar el bienestar de otro (Eisenberg, Fabes y Spinrad, 2006). Actuando autónomamente, la prosocialidad no solo contribuye al bienestar del que recibe la acción y mejora las relaciones interpersonales (Caputi, Lecce, Pagnin y Banerjee, 2012; Weinstein y Ryan, 2010), sino que además genera afecto positivo y bienestar en la persona que realiza tales conductas (Dunn, Aknin y Norton, 2008; Steger, Kashdan y Oishi, 2008).

Diferentes autores, consideran la conducta prosocial como protector de los estilos de interacción social desadaptativos, tales como la agresividad, el aislamiento o el retraimiento social (Eisenberg et al., 2006; Gilma y Anderman, 2006; Inglés, Martínez-González y GarcíaFernández, 2013; Veenstra et al., 2008; Wentzel, 2005) desempeñando un papel importante en la formación de relaciones interpersonales positivas y en la aceptación por los iguales, padres y profesores (Eisenberg et al., 2006; Gilman y Anderman, 2006; Inglés, Delgado, García, Ruiz y Díaz, 2010), siendo un buen indicador de la adaptación escolar y social del alumnado (Guevara, Cabrera y Barrera, 2007).

En el estudio de la relevancia del desarrollo del hábito de la actividad física a edades tempranas, las investigaciones en el área de la educación (Cecchini, Fernández, González y Arruza, 2008) muestran que mediante el juego y la práctica deportiva, se consiguen aprender y/o mejorar competencias esenciales como el autocontrol, la empatía, el autoconcepto o los comportamientos prosociales, y añaden que son transferibles a otros ámbitos como la familia o la escuela, por lo que es considerada un elemento muy a tener en cuenta.

Cuando se ha señalado su relación con respecto al género, se ha concluido que las chicas presentan niveles significativamente más altos de conducta prosocial que los chicos, aumentando estas diferencias con la edad (Álvarez, Carrasco y Fustos, 2010; Carlo et al., 2013; Inglés et al., 2009; Kärtner, Keller y Chaudhary, 2010; Kumru, Carlo, Mestre y Samper, 2012; Plazas et al., 2010). Al mismo tiempo, los comportamientos prosociales de los adolescentes en la práctica social del deporte (inter e intragénero), mantienen una relación estrecha 
con la autorregulación emocional (Richaud y Mesurado, 2016; Torstveit, Sütterlin y Lugo, 2016), siendo el principal protector de conductas agresivas, antisociales y comportamientos inadaptados (Carlo et al., 2014; Kornbluh y Neal, 2016; Mesurado et al., 2014; Mikolajewski, Chavarria, Moltisanti, Hart y Taylor, 2014).

Algunas investigaciones, han observado la asociación positiva entre los comportamientos sociales y la práctica de actividad física en el contexto educativo y fuera del mismo (González y Portolés, 2016; Samadzadeh, Abbasi y Shahbazzadegan, 2011), así como los vínculos emocionales positivos o negativos que se establecen entre iguales y docentes (Jiménez, Estévez y Murgui, 2014; Samper, Mestre y Malonda, 2015). Tales vivencias en la adolescencia, establecen una conexión cognitiva con valores personales y normas que subyacen en el desarrollo psicosocial, favoreciendo el desarrollo de actitudes prosociales (Revuelta y Esnaola, 2011). La actividad física se considera una herramienta muy útil para la obtención de estos aprendizajes, y el centro educativo un lugar en el que se pueden consolidar, junto con otros elementos del crecimiento de los adolescentes (González, Caprara, Garcés de los Fayos y Zuffianó, 2014).

\section{Objetivos e hipótesis}

Teniendo en cuenta todas estas premisas, se diseña y expone el presente trabajo con los objetivos de: a) establecer diferencias entre variables internas (autoconcepto y prosocialidad) y externas (freciencia de actividad física) que rodean al adolescente en el contexto escolar, según condiciones sociales (sexo, tipo de práctica de actividad física y edad); y b) proponer relaciones de causalidad y predictibilidad entre las diferentes dimensiones del autoconcepto y conducta prosocial hacia la práctica de actividad física en una muestra de adolescentes.

\section{Método}

\section{Participantes}

En el presente estudio se ha llevado a cabo una metodología de carácter transversal e incidental, en una muestra de estudiantes de Educación Secundaria Obligatoria y Bachillerato, de tres centros educativos ubicados en la Región de Murcia. La muestra invitada fue de 486 estudiantes de $1^{\circ}$ de ESO a $2^{\circ}$ de Bachillerato. De ellos, finalmente participaron 432, de los que $23(5.32 \%)$ fueron excluidos por no obtener el consentimiento informado de los padres o por presentar errores u omisiones en sus respuestas. Por tanto, la muestra real fue de 409 participantes $(n=409)$, distribuidos por nivel académico en $1^{\circ}$ de la ESO $(n=45 ; 11.0 \%), 2^{\circ}$ de 
la ESO $(\mathrm{n}=84 ; 20.5 \%), 3^{\circ}$ de la ESO $(\mathrm{n}=76 ; 18.6 \%), 4^{\circ}$ de la ESO $(\mathrm{n}=69 ; 16.9 \%), 1^{\mathrm{o}}$ de Bachillerato $(\mathrm{n}=86 ; 21.0 \%)$, y $2^{\circ}$ de Bachillerato $(\mathrm{n}=49 ; 12.0 \%)$. En cuanto a la variable género, 185 de los participantes son chicos (45.2\%) y 224 chicas $(54.8 \%)$, encontrándose en una edad comprendida entre los 12 y los 19 años $(\mathrm{M}=14.81$; DT =1.79).

Centrándonos ahora en las variables que tienen relación con la práctica de actividad física, la distribución de la muestra se presenta en práctica actividad física $(\mathrm{n}=309 ; 75.6 \%)$ y no practica actividad física $(\mathrm{n}=100 ; 24.4 \%)$. Además, 92 participantes indican estar federados (22.5\%), y 317 no federados (77.5\%). Si se analiza de forma pormenorizada, en relación a la edad de los participantes (Figura 1), los chicos realizan actividad física con una frecuencia media de 3.5 días a la semana, mientras que las chicas lo hacen 2.18 días.

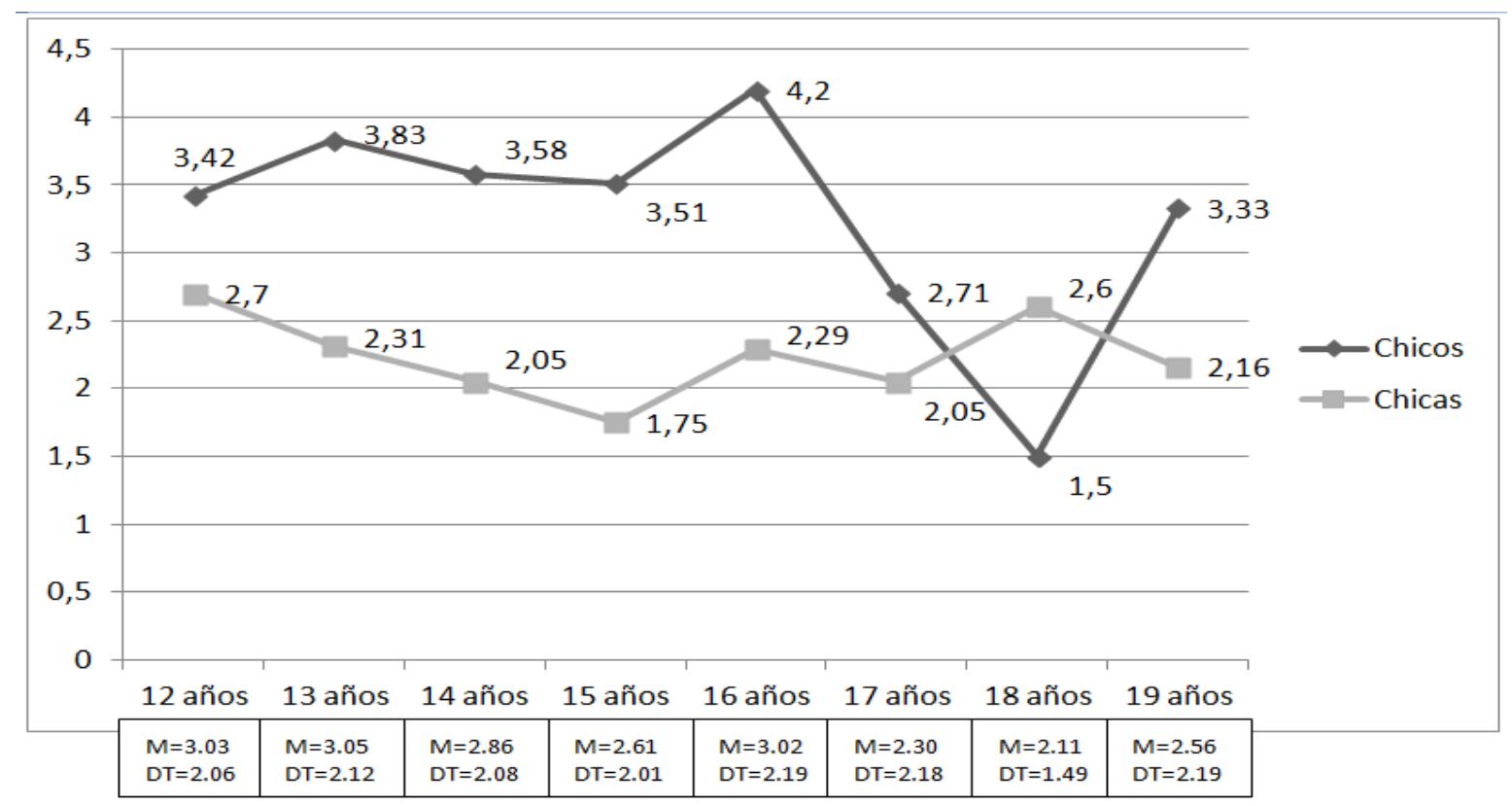

Figura 1. Días de práctica de actividad física de los participantes, en relación a la edad.

Del mismo modo, los adolescentes indican los contextos donde más fomentan sus relaciones sociales. Como se puede apreciar en la tabla 1, cerca de la mitad de los estudiantes que forman parte de la muestra, señalan que el principal ámbito donde fomentan sus relaciones sociales es el de los amigos (42.3\%), seguido de la escuela (34\%, y 36.7\% si es tercera opción), señalando el deporte como el entorno menos propicio de los cuatro para fomentar sus relaciones sociales $(42.1 \%)$. La familia se mantiene en una posición intermedia dentro de sus prioridades. 
Tabla 1. Importancia de los contextos para el fomento de relaciones sociales de los participantes.

\begin{tabular}{lccccc}
\hline $\mathrm{N}=409$ & & Fomento Rel. Soc.1 & Fomento Rel. Soc.2 & Fomento Rel. Soc.3 & Fomento Rel. Soc.4 \\
& Deporte & $75(18.3 \%)$ & $59(14.4 \%)$ & $101(24.7 \%)$ & $\mathbf{1 7 2}(\mathbf{4 2 . 1 \% )})$ \\
& Familia & $89(21.8 \%)$ & $96(23.5 \%)$ & $105(25.7 \%)$ & $119(29.1 \%)$ \\
& Escuela & $72(17.6 \%)$ & $\mathbf{1 3 9}(\mathbf{3 4 \%})$ & $\mathbf{1 5 0}(\mathbf{3 6 . 7 \% )}$ & $50(12.2 \%)$ \\
Amigos & $\mathbf{1 7 3 ( 4 2 . 3 \% )}$ & $115(28.1 \%)$ & $53(13.0 \%)$ & $68(16.6 \%)$ \\
\hline
\end{tabular}

Atendiendo a la relación del alumnado por curso y género (Figura 2), la frecuencia de actividad física media está comprendida entre 2.26 y 4.33 días a la semana, por parte de los chicos, y entre 1.85 y 3.37 veces por semana si se hace referencia a las chicas. Además, también se puede comprobar que el porcentaje de práctica de actividad física por parte de los chicos supera ampliamente al de las chicas en todos los cursos académicos excepto en $2^{\circ}$ de Bachillerato, que prácticamente están equiparados. Del mismo modo, se aprecia que conforme aumenta la edad, tanto chicos como chicas tienden a realizar menos actividad física, pasando de casi el $100 \%$ en $1^{\circ} \mathrm{ESO}$, por parte de los chicos, hasta poco más de la mitad en el último curso estudiado.

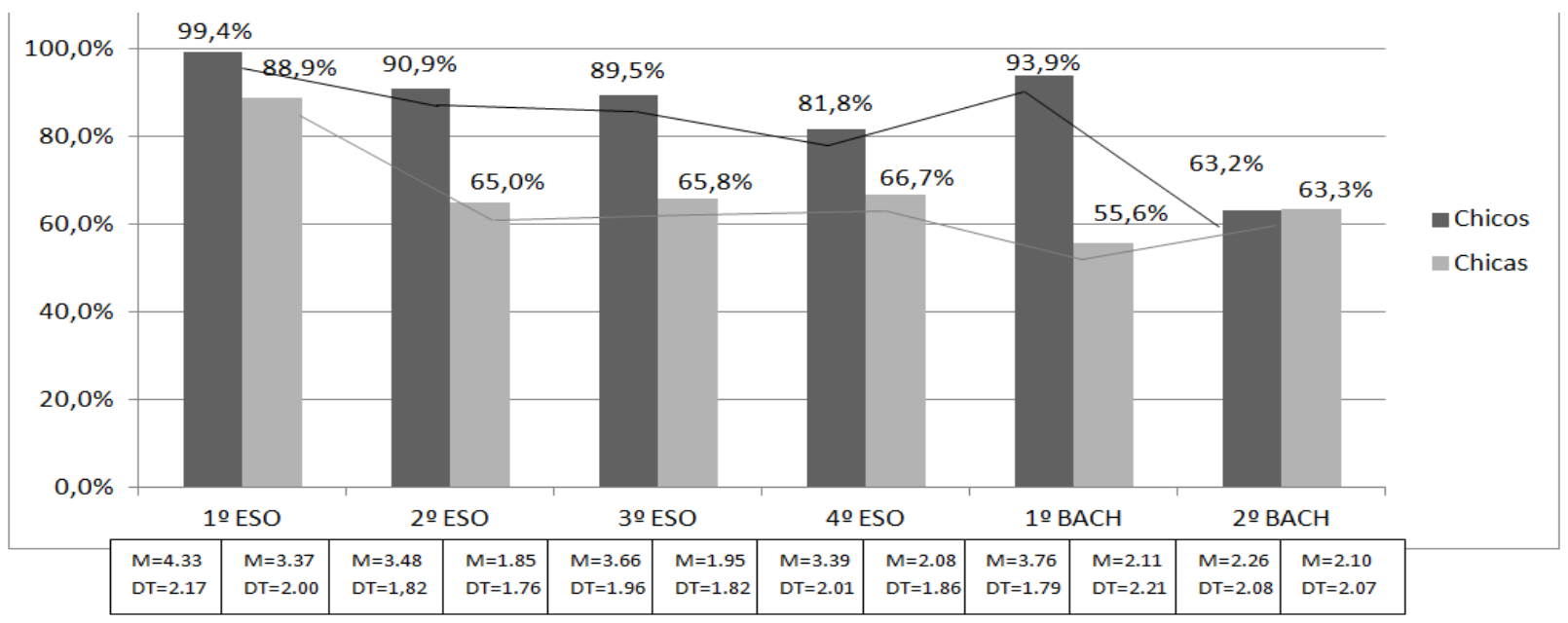

Figura 2. Práctica de actividad física, distribuida a través del género y cursos de los participantes.

Si se realiza esta misma comparación en relación a la conducta prosocial (Figura 3), se observa cómo el nivel medio de prosocialidad de las chicas supera al de los chicos en todos los cursos académicos estudiados, obteniendo la máxima diferencia entre ambos sexos en $4^{\circ}$ ESO. 


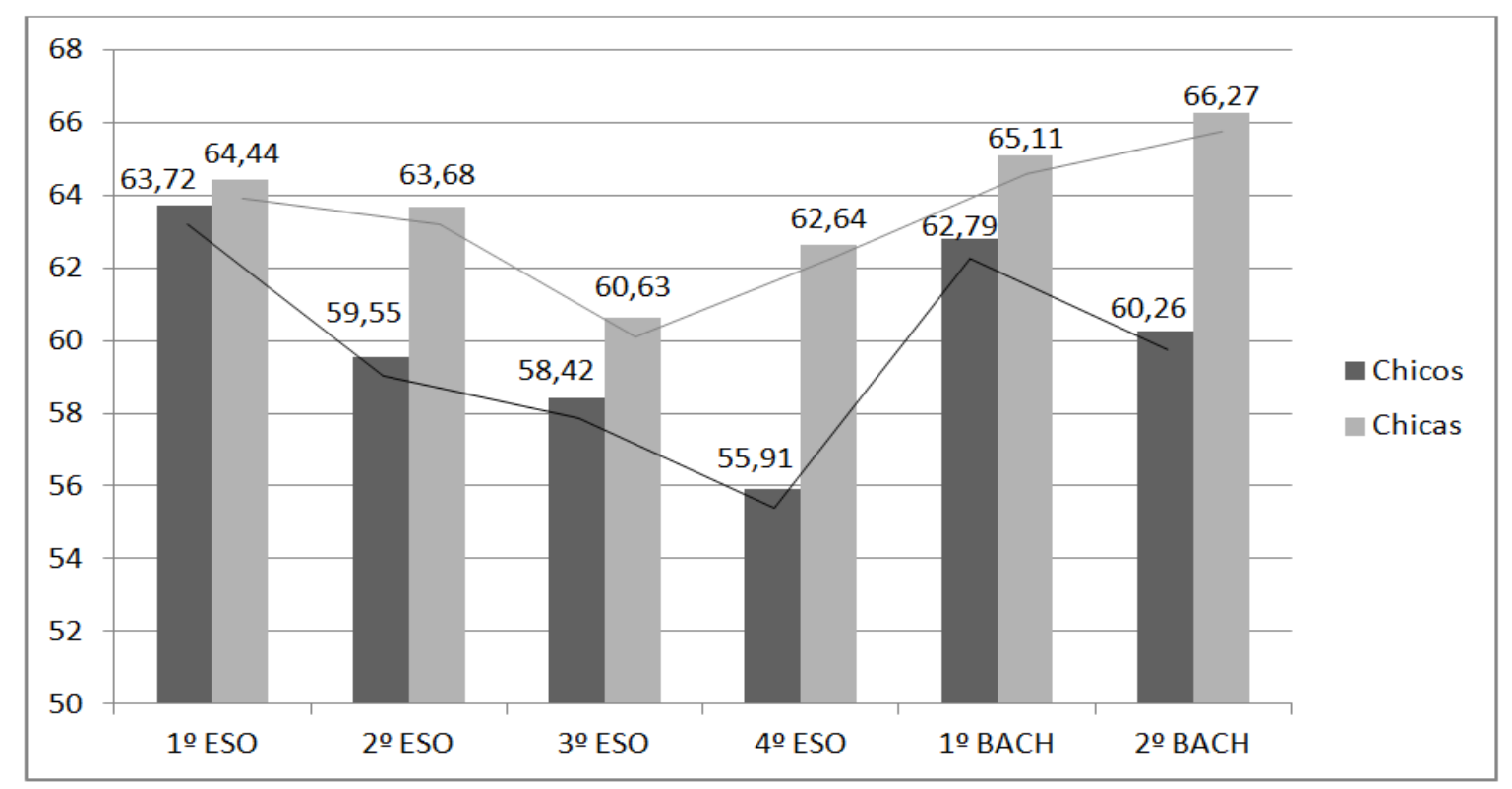

Figura 3. Nivel medio de prosocialidad, distribuida por curso y género de los participantes.

\section{Instrumentos}

Sociodemográfico. Se confecciona un autoinforme "ad hoc" con una serie de ítems mediante los cuales se tienen en cuenta la edad, sexo, práctica de actividad física y frecuencia, condición de deportista federado y modalidad deportiva, cantidad de hermanos y entornos donde los participantes fomentan más sus relaciones sociales.

Autoconcepto. Para medir el autoconcepto ha sido empleada la $4^{\mathrm{a}}$ edición del Cuestionario de Autoconcepto (AF-5; García y Musitu, 2014). Parte de una consideración multidimensional del autoconcepto, incluyendo ítems que permiten evaluar el concepto y la valoración que el sujeto tiene de sí mismo en cinco dimensiones personales y relacionales: académica, social, emocional, familiar y física. El cuestionario consta de 30 ítems con una orientación de respuesta de 1 a 99, mostrando una fiabilidad interna alpha de.77.

Prosocialidad. Escala de prosocialidad en adolescentes y adultos (Caprara, Steca, Zelli e Capanna, 2005. Adaptación al español): la cual se compone de 16 ítems con escala Likert de 5 puntos ( 1 a 5$)$. Cada ítem se evalúa en una escala de 5 posiciones $(1=$ casi nunca; $2=$ pocas veces; 3 = alguna vez; 4 = muchas veces 5 = casi siempre/siempre). La escala para adolescentes y adultos refleja en parte la extensión, a una población más adulta, haber desarrollado de una manera más adecuada para una población de adultos los ítems relativos a los tres compo- 
nentes de la conducta pro-social (ayudar, cuidar y compartir) se añadieron cuatro afirmaciones para la detección de habilidades cognitivo-afectivas que permiten a los individuos ponerse en la perspectiva del otro y comprender la llamada de ayuda, o sea la empatía. La consistencia interna del instrumento para la presente muestra, es muy confiable con un alpha de .87 , y se compone de 16 ítems para evaluar con qué frecuencia las personas tienden a compartir las cosas propias con los demás, cuidar a los demás, entender cuando alguien necesita ayuda y da asistencia.

Dificultades para la socialización: Escala de Dificultades de Socialización de Cantoblanco (SOC; Herrero, Escorial y Colom, 2009). Este instrumento (previamente conocido como Escala de Dificultades de Temperamento de Cantoblanco) es un autoinforme de 45 ítems que evalúa tres rasgos de personalidad: impulsividad, ausencia de miedo y búsqueda de sensaciones. Estos rasgos se señalan en la interpretación sobre el comportamiento antisocial. Cada ítem plantea dos opciones de respuesta en forma de dos situaciones entre las que se ha de elegir. Una de las opciones refleja presencia del rasgo, mientras que la otra es sintomática de ausencia. En las subescalas de impulsividad y búsqueda de sensaciones, una de las opciones es una situación o actividad que implica un alto nivel de esos rasgos, frente a otra en la que se plantea una situación de bajo nivel. Un ejemplo de ítem de búsqueda de sensaciones es elegir entre "Ir a un concierto de música clásica o Tirarte de un puente atado a una cuerda elástica”. En el caso de la subescala de ausencia de miedo se planteaba una situación que implicaba una alta peligrosidad frente a otra que era muy molesta. El razonamiento era que una persona con un bajo miedo preferiría la situación peligrosa a la molesta. Un ítem de la subescala es elegir entre "Perder la cartera con mucho dinero y la documentación o enzarzarte en una pelea con un desconocido”. A modo de rasgo general, se define también la dimensión de dificultades para la socialización. El instrumento ha mostrado índices de consistencia interna de .79 .

\section{Procedimiento}

Los datos se han recogido durante el curso académico 2016-2017, estableciendo el primer contacto presencial con los centros educativos a finales del primer trimestre. Para dar inicio al proceso, se lleva a cabo una reunión individualizada con los directores de cada uno de los centros y se les informa sobre la temática y el objeto del estudio que se quiere desarrollar, solicitando su colaboración para realizar la investigación. Obtenido el permiso oportuno, se estableció la mejor área para recoger los datos en cada uno de los centros y se pasó a hablar 
con el profesorado afectado con la intención de darle a conocer el principal objetivo de este estudio, así como pedir su colaboración. Tras esto, se informa al alumnado del trabajo y se le proporciona el consentimiento informado para que lo aprueben sus padres-tutores (Helsinki, 2008). Aceptada la propuesta, se fijaron las fechas en las que se iba a realizar la prueba en cada uno de los centros y en cada una de las clases partícipes, algunas elegidas por conveniencia en cuanto a horarios, y en otras al azar. A partir de entonces, se procedió a la recogida de datos en diferentes días y sesiones, siempre en presencia del docente de la asignatura y del investigador principal.

\section{Análisis de datos}

Se llevan a cabo análisis estadísticos básicos que permitan la descripción de la muestra, medidas de tendencia central, de frecuencias, de homogeneidad y de fiabilidad interna de los instrumentos. De la misma forma, y para la diferenciación intermuestral (ANOVA y pruebas $t$ de diferencias de medias). Para determinar la relación entre las variables objeto de estudio, se realizan las relaciones de causalidad a través de análisis de relaciones causales entre las variables mediante análisis de correlación bidireccional. El programa estadístico utilizado es el SPSS (IBM), en su versión 23.

\section{Resultados}

Los análisis llevados a cabo tratan de establecer y explicar la interrelación de las variables, así como el contraste diferencial para las variables sociodemográficas que se han tenido en consideración.

\section{Análisis diferenciales}

Atendiendo a las relaciones diferenciales en la variable género (Tabla 2), se hacen relevantes las diferencias significativas en prosocialidad $\left(t_{2,407}=-4.32 ; p<.00\right)$, a favor de las chicas, y en autoconcepto físico $\left(t_{2,407}=5.63 ; p<.00\right)$ a favor de los chicos, aunque también cabe destacar los bajos niveles de autoconcepto emocional en ambos sexos. En el resto de las dimensiones relacionadas con el autoconcepto (académico, social, familiar y general) no se encuentran diferencias significativas cuando se comparan chicos y chicas.

En relación a la variable práctica de actividad física, se puede observar que 309 (75.6\%) participantes del total de la muestra realizan actividad física al menos un día a la se- 
mana, mientras que $100(24.4 \%)$ de los adolescentes no acostumbran a practicar actividad física en su día a día. En este sentido, los resultados señalan importantes diferencias significativas a favor de los practicantes de actividad física en la mayor parte de las dimensiones del autoconcepto, destacando el autoconcepto físico $\left(t_{2,407}=7.06 ; p<.00\right)$ y el autoconcepto general $\left(t_{2,407}=4.07 ; p<.00\right)$. En el caso contrario, las únicas diferencias significativas a favor de los no practicantes de actividad física aparecen en el autoconcepto emocional $\left(t_{2,407}=-\right.$ 2.23; $p<.03$ ), no apareciendo diferencias significativas en dimensiones relacionadas con las relaciones sociales como prosocialidad o dificultades de socialización.

En cuanto a la variable federado, aparecen diferencias significativas en la mayor parte de las dimensiones del autoconcepto, destacando el autoconcepto físico $\left(t_{2,407}=8.05 ; p<.00\right)$ y autoconcepto social $\left(t_{2,407}=2.24 ; p<.03\right)$ a favor de los federados, y el autoconcepto emocional $\left(t_{2,407}=-3.00 ; p<.00\right)$ a favor de los no federados; no encontrando diferencias significativas cuando se comparan en la dimensión académica. Además, aparecen diferencias significativas en las dimensiones ausencia de miedo $\left(t_{2,407}=-3.46 ; p<.00\right)$ y dificultades de socialización $\left(t_{2,407}=-2.96 ; p<.00\right)$ para los participantes que no están federados en ninguna disciplina deportiva, y no se han encontrado en prosocialidad, impulsividad y búsqueda de sensaciones.

Tabla 2. Diferencias, según las variables práctica de actividad física y género.

\begin{tabular}{|c|c|c|c|c|c|c|c|c|}
\hline & & \multicolumn{2}{|c|}{$\mathrm{M}(\mathrm{DT})$} & $\mathrm{F}$ & Sig. & $\mathrm{T}$ & $\mathrm{Gl}_{(\mathrm{n}-2)}$ & Sig. \\
\hline \multirow{7}{*}{$\begin{array}{l}\text { Practica AF } \\
(n=309) \\
\text { No practica } \\
\text { AF }(n=100)\end{array}$} & & $\mathbf{A F}$ & NO AF & & & & \multirow{6}{*}{407} & \\
\hline & AUT. ACADEMICO & $6.95(1.82)$ & $6.32(2.01)$ & 2.06 & .15 & 2.91 & & .00 \\
\hline & AUT.SOCIAL & $7.50(2.07)$ & $6.90(1.78)$ & .11 & .75 & 2.60 & & .01 \\
\hline & AUT.EMOCIONAL & $4.59(2.20)$ & $5.14(1.99)$ & .41 & .52 & -2.23 & & .03 \\
\hline & AUT.FISICO & $6.60(1.96)$ & $4.98(2.05)$ & .57 & .45 & 7.06 & & .00 \\
\hline & AUT.GENERAL & $6.80(1.12)$ & $6.27(1.12)$ & .18 & .68 & 4.07 & & .00 \\
\hline & & Federado & No Federado & & & & & \\
\hline \multirow{8}{*}{$\begin{array}{l}\text { Federados } \\
(\mathrm{n}=92) \\
\text { No federa- } \\
\text { dos }(\mathrm{n}=317)\end{array}$} & AUT.SOCIAL & 7.76(1.95) & $7.23(2.02)$ & .11 & .74 & 2.24 & \multirow[t]{7}{*}{407} & .03 \\
\hline & AUT.EMOCIONAL & $4.13(1.78)$ & $4.89(1.78)$ & 2.67 & .10 & -3.00 & & .00 \\
\hline & AUT.FAMILIAR & $8.64(1.48)$ & $8.17(2.14)$ & 3.38 & .07 & 1.93 & & .05 \\
\hline & AUT.FISICO & $7.64(1.57)$ & $5.78(2.05)$ & 9.16 & .00 & 8.05 & & .00 \\
\hline & AUT.GENERAL & $7.04(.90)$ & $6.56(1.18)$ & 4.12 & .04 & 3.56 & & .00 \\
\hline & $\begin{array}{l}\text { AUSENCIA MIE- } \\
\text { DOS }\end{array}$ & $22.66(1.57)$ & $23.15(1.77)$ & 1.89 & .17 & -3.46 & & .00 \\
\hline & D.SOCIALIZACION & $22.65(.96)$ & $22.98(.92)$ & .49 & .48 & -2.96 & & .00 \\
\hline & & Chicos & Chicas & & & & & \\
\hline chicos & PROSOCIALIDAD & $59.72(9.57)$ & $63.77(9.57)$ & .51 & .48 & -4.32 & 407 & .00 \\
\hline$(n=185)$ & AUT.EMOCIONAL & $4.29(2.03)$ & $5.09(2.19)$ & .26 & .61 & -3.80 & & .00 \\
\hline chicas & AUT.FISICO & $6.82(2.01)$ & $5.69(2.05)$ & .14 & .70 & 5.63 & & .00 \\
\hline
\end{tabular}




\begin{tabular}{|c|c|c|c|c|c|c|c|}
\hline$(n=224)$ & IMPULSIVIDADES & $22.14(1.62)$ & $22.54(1.59)$ & .04 & .85 & -2.53 & .01 \\
\hline
\end{tabular}

Tabla 3. Análisis de correlaciones entre las variables estudiadas.

\section{Análisis relacionales}

Al analizar la relación existente entre las variables estudiadas (Tabla 3), son numerosas las correlaciones significativas que se ponen de manifiesto. De entre ellas, se hace relevante destacar la correlación directa y significativa entre la frecuencia de actividad física y el autoconcepto físico $(r=.41 ; p<.00)$, de tal forma que conforme aumenta la frecuencia de actividad física, incrementa el autoconcepto físico. De la misma manera, el autoconcepto académico también correlaciona directa y significativamente con el autoconcepto físico $(r=.37$; $p<.00)$.

Por otra parte, la prosocialidad muestra una correlación directa y significativa con el autoconcepto general $(r=.21 ; p<.00)$, de manera que cuanto mayor es la conducta prosocial, mejor percepción tiene esa persona de sí mismo. Del mismo modo, ocurre al correlacionar el autoconcepto emocional con la ausencia de miedo $(r=.19 ; p<.00)$.

Por último, otra de las relaciones que conviene mencionar, es la que se observa entre la frecuencia de actividad física y ausencia de miedo, puesto que señala una correlación inversa y significativa $(r=-.10 ; p<.04)$, de forma que cuanto mayor es la frecuencia con la que se realiza actividad física, los adolescentes encuestados señalan disponer de mayores indicadores de temor ante situaciones amenazantes.

En otro orden, el modelo predictivo (Tabla 4) señala, con un $20,3 \%$ de varianza explicada $\left(F_{400,9)}=11.29 ; p<.00\right)$, que la práctica de actividad física aumenta conforme aumenta la prosocialidad $(\beta=.45 ; p<.04)$, el autoconcepto social $(\beta=.61 ; p<.04)$, físico $(\beta=.73 ; p$ $<.00)$ y emocional $(\beta=.63 ; p<.00)$, así como la búsqueda de sensaciones $(\beta=.54 ; p<.03)$. 


\begin{tabular}{|c|c|c|c|c|c|c|c|c|c|c|c|c|c|}
\hline $\mathrm{N}=409$ & 1 & 2 & 3 & 4 & 5 & 6 & 7 & 8 & 9 & 10 & 11 & 12 & 13 \\
\hline 1. CURSO & 1 & $-.14^{* *}$ & .06 & -.07 & $-.12^{*}$ & .04 & -.07 & $-.12^{*}$ & $-.12^{*}$ & -.09 & -.03 & -.05 & -.09 \\
\hline 2. FRECAF & & 1 & .05 & $.10^{*}$ & $.18^{* *}$ & $-.16^{* *}$ & .05 & $.41^{* *}$ & $.20^{* *}$ & .03 & $-.10^{*}$ & .01 & -.04 \\
\hline 3. PROSOCIALIDAD & & & 1 & $.20^{* *}$ & $.16^{* *}$ & .10 & .07 & .06 & $.21^{* *}$ & -.05 & $.18^{* *}$ & $.12^{*}$ & $.16^{* *}$ \\
\hline 4. AUTOCONCEPTO ACADEMICO & & & & 1 & $.16^{* *}$ & -.07 & $.34^{* *}$ & $.37^{* *}$ & $.62^{* *}$ & -.06 & .06 & $.16^{* *}$ & $.10^{*}$ \\
\hline 5. AUTOCONCEPTO SOCIAL & & & & & 1 & $-.15^{* *}$ & $.29^{* *}$ & $.41^{* *}$ & $.60^{* *}$ & .07 & -03 & -03 & -01 \\
\hline 6. AUTOCONCEPTO EMOCIONAL & & & & & & 1 & -.09 & -.08 & $.24^{* *}$ & -.02 & $.19^{* *}$ & .08 & $.15^{* *}$ \\
\hline 7. AUTOCONCEPTO FAMILIAR & & & & & & & 1 & $.32^{* *}$ & $.65^{* *}$ & -.07 & .06 & .06 & .03 \\
\hline 8. AUTOCONCEPTO FISICO & & & & & & & & 1 & $.71^{* *}$ & .03 & -.09 & -.01 & -.05 \\
\hline 9. AUTOCONCEPTO GENERAL & & & & & & & & & 1 & -.02 & .07 & .09 & .08 \\
\hline 10. BUSQUEDA SENSACIONES & & & & & & & & & & 1 & -.01 & $-.16^{* *}$ & $.43^{* *}$ \\
\hline 11. AUSENCIA MIEDOS & & & & & & & & & & & 1 & $.14^{* *}$ & $.70^{* *}$ \\
\hline 12. IMPULSIVIDADES & & & & & & & & & & & & 1 & $.58^{* *}$ \\
\hline 13. DIFICULTAD SOCIALIZADORA & & & & & & & & & & & & & 1 \\
\hline
\end{tabular}

$* p<.05 \quad * * p<.01$

Tabla 4. Modelo predictivo de la actividad física, en función de la prosocialidad, autoconcepto y dificultades de socialización.

\begin{tabular}{lccccc}
\hline $\left.\mathrm{R}^{2}=.230 ; \mathrm{F}_{(400,9)}=11.29 ; \mathrm{p}<.00\right)$ & $\mathrm{B}$ & Error típ. & Beta & $\mathrm{F}$ & $\mathrm{p}$ \\
$\mathrm{N}=409$ & .74 & 2.43 & & .30 & .76 \\
\hline Constante) & $\mathbf{. 4 1}$ & $\mathbf{. 0 1}$ & $\mathbf{. 4 5}$ & $\mathbf{1 . 1 0}$ & $\mathbf{. 0 4 *}$ \\
PROSOCIALIDAD & -.06 & .05 & -.05 & -1.06 & .28 \\
AUTOCONCEPTO ACADEMICO & $\mathbf{. 2 0}$ & $\mathbf{. 0 5}$ & $\mathbf{. 6 1}$ & $\mathbf{. 1 7}$ & $\mathbf{. 0 4 *}$ \\
AUTOCONCEPTO SOCIAL & $\mathbf{- . 3 3}$ & $\mathbf{. 0 4}$ & $\mathbf{. . 6 3}$ & $\mathbf{2 . 9 2}$ & $\mathbf{. 0 0 * *}$ \\
AUTOCONCEPTO EMOCIONAL & -.09 & .05 & -.08 & -1.76 & .09 \\
AUTOCONCEPTO FAMILIAR & $\mathbf{. 4 3}$ & $\mathbf{0 5}$ & $\mathbf{. 7 3}$ & $\mathbf{8 . 2 8}$ & $\mathbf{. 0 0 * *}$ \\
AUTOCONCEPTO FISICO & $\mathbf{. 5 6}$ & $\mathbf{. 0 8}$ & $\mathbf{. 5 4}$ & $\mathbf{. 7 7}$ & $\mathbf{. 0 3}$ \\
BUSQUEDA SENSACIONES & .09 & .08 & .07 & 1.14 & .25 \\
IMPULSIVIDADES & -.14 & .16 & -.06 & -.88 & .37 \\
DIFICULTAD SOCIALIZADORA & & & & &
\end{tabular}

Variable dependiente: FRECAF

\section{Discusión y Conclusiones}

El objetivo del presente trabajo se dirige a contemplar las relaciones estadísticas que aparecen en una muestra de adolescentes sobre sus recursos prosociales y autopercepción individual en cuestión de su género, práctica de actividad física que realizan y la edad. Por lo general, la literatura se centra más en los efectos que produce el ejercicio en la salud física, 
que los que puede generar en la salud psicológica (González et al., 2014), o en la influencia social de esta a edades tempranas. Es por ello, que el trabajo constata las tendencias hacia la realización de práctica de actividad física en los adolescentes, desde el punto de vista de su percepción social como individuos que se desenvuelven dentro del contexto escolar.

A tenor de los resultados alcanzados, cabe destacar que un importante número de los participantes que han formado parte de la muestra (75.6\%) realizan actividad física al menos un día a la semana, lo cual se aproxima a los hallazgos encontrados en otros estudios como el de González y Portolés (2014), que sitúan al alumnado practicante de actividad física en el $80.5 \%$. Además, resulta relevante comentar que, entre los estudiantes que realizan actividad física, los chicos (3.5) practican mayor número de días a la semana que las chicas (2.18), asemejándose a los resultados obtenidos por Alvariñas, Fernández y López (2009), que establecen una práctica de 4 o 5 veces a la semana por parte de los chicos y 2 o 3 días por parte de las chicas.

La disminución de la frecuencia de práctica de actividad física conforme aumenta la edad de los participantes, se hace evidente al pasar de 3.85 días en el alumnado de $1^{\circ}$ ESO a 2.18 días en el de $2^{\circ}$ Bachillerato. Esto corrobora lo que indican otros estudios (González y Portolés, 2014; Ruiz-Juan y García, 2002) que afirman que las mayores tasas de actividad física y deporte se producen entre los más jóvenes, reduciéndose al incrementarse la edad.

Por otra parte, los participantes señalan que el contexto social más influyente para el fomento de sus relaciones sociales es el de los amigos, mientras que la familia queda relegada a un segundo plano. Este hecho confirma lo que argumentan numerosos estudios (Janosz, Archambault, Morizot y Pagan, 2008; Li, Doyle, Kalvin, Liu y Lerner, 2011; Martínez et al., 2010; Vanatta, Gartsein, Zeller y Noll, 2009), que observan en los adolescentes un incremento significativo de la influencia del grupo de iguales, siendo el contexto de socialización más influyente, en detrimento del familiar, que se va reduciendo durante este periodo.

Los resultados de este estudio indican que las chicas presentan niveles de conducta prosocial significativamente más altos que los chicos, confirmando lo que han demostrado numerosas investigaciones (Álvarez, Carrasco y Fustos, 2010; Carlo et al., 2013; Inglés et al., 2009; Kärtner, Keller y Chaudhary, 2010; Kumru, Carlo, Mestre y Samper, 2012; Plazas et al., 2010). Además, resulta expresivo que aparezca una relación positiva y significativa con 
dificultades de socialización, debido a que algunos autores (Eisenberg et al., 2006; Gilman y Anderman, 2006; Inglés et al., 2010) señalan que desempeña un papel fundamental en la formación de relaciones interpersonales positivas y en la aceptación por los iguales, siendo incluso un buen indicador de la aceptación escolar y social del alumnado (Guevara et al., 2007).

En otro orden, los resultados muestran que la conducta prosocial también tiene una relación directa y significativa con el autoconcepto general, y con sus dimensiones académica y social. Esto respalda los hallazgos encontrados por Inglés et al. (2013), quienes relacionan la conducta prosocial con el autoconcepto escolar y, además, la consideran un factor clave para la competencia social y académica del alumnado.

Atendiendo a las diferentes dimensiones del autoconcepto, los chicos presentan niveles de aucoconcepto físico significativamente más altos que las chicas, confirmando lo que argumentan estudios como Páez, Fachinnelli, Gutiérrez-Martínez y Hernández (2007). En cambio, se ha comprobado que existen diferencias significativas en la dimensión del autoconcepto emocional a favor de las chicas con respecto a los chicos. Esto difiere de lo que indican autores como Padilla, García y Suárez (2010), quienes señalan que aunque existe una tendencia de los chicos a tener mejor imagen de sí mismos en los ámbitos físico, emocional y social, las diferencias con respecto a las chicas solo son significativas en el autoconcepto emocional y en el autoconcepto global no académico a favor de los chicos.

Continuando con el estudio del autoconcepto, se hace relevante comentar la aparición de diferencias significativas en las dimensiones del autoconcepto físico, social y general a favor de los participantes federados; así como una tendencia a la significación en el autoconcepto familiar. Por el contrario, en autoconcepto emocional, ausencia de miedo y dificultades de socialización, las diferencias se postulan a favor de los adolescentes que no practican actividad física de forma federada. Los resultados aquí expuestos, corresponden en gran medida con los de Martínez y González (2017), quienes afirman encontrar niveles de autoconcepto físico significativamente mayores en adolescentes federados, así como en ausencia de miedo, impulsividad y dificultades de socialización a favor de los no federados. Del mismo modo, se observa una relación causal significativa entre el autoconcepto familiar, con el físico y el general. 
Por otra parte, numerosas investigaciones confirman una relación entre el autoconcepto físico y el nivel de actividad físico-deportiva (Aróstegi, Goñi, Zubillaga e Infante, 2012; Esnaola y Zulaika, 2009; Revuelta y Esnaola, 2011; Zalagaz, Castro, Valdivia y Cachón, 2017), indicando incluso que esta conexión aumenta en quienes practican actividad física con mayor frecuencia (Goñi e Infante, 2010). Los datos aquí obtenidos refuerzan estos hallazgos, al destacar la relación directa y significativa que se manifiesta entre el autoconcepto físico y general con la frecuencia de actividad física.

A modo de conclusión, se puede indicar que la práctica habitual de actividad física en los adolescentes puede ser una herramienta educativa muy útil para trabajar y/o mejorar tanto el autoconcepto como las conductas prosociales. Esto desemboca en un incremento en la confianza en uno mismo, reduciendo sus dificultades para establecer relaciones sociales con el resto de compañeros, siendo más atrevidos como consecuencia, y mejorando su integración, no sin esfuerzo, dentro del grupo-clase.

Para ello, se considera necesario luchar contra aspectos como la poca valoración social del deporte femenino en la sociedad, o la orientación a la extra práctica del deporte federado frente a la práctica de ejercicio como modelo de ocupación del tiempo libre en los adolescentes, pues no generan elementos de implicación social o de autovaloración personal positiva, que faciliten la percepción de utilidad de la práctica de actividad física como hábito en sus vidas cotidianas. Esto hace que resulte muy complicado que las chicas se sientan lo suficientemente estimuladas por el ejercicio, o los no federados practiquen deporte libre en general, sin la pertenencia a entornos más estructurados como clubes o federaciones.

Por otra parte, resulta curiosa la poca influencia que genera la familia entre los adolescentes como contexto donde fomentar sus relaciones sociales, a pesar de que es el grupo social primario por excelencia. Desde esta óptica, la edad de los participantes se considera un factor de peso para explicar el motivo de estos resultados, por lo que podría ser interesante seguir investigando esta cuestión con participantes que se encuentren en una ratio de edad diferente. Además, también se hace necesaria la profundización de estudios sobre las relaciones prosociales y la respuesta de dificultades para la socialización, de manera que sea posible explicar cómo la tendencia a ser social y colaborativo, se relaciona directamente con la respuesta impulsiva desde el punto de vista social, y con la consideración de menores recursos y habilidades para la consolidación de las relaciones sociales. 
En cuanto a las limitaciones del estudio, destaca sobremanera las dificultades que existen hoy en día para acceder a la muestra debido a que los centros educativos están saturados. Cada año son más los estudiantes universitarios e investigadores que solicitan sus dependencias y resulta muy complicado conseguir el permiso para poder proceder a la recogida de datos. Además, al plantearse como una actividad voluntaria que no interfiere en los resultados académicos, el alumnado suele olvidar el consentimiento informado cumplimentado por sus tutores legales, por lo que la demora temporal y la pérdida de participantes durante el proceso se hacen evidentes. Otra de las limitaciones que se pueden plantear sobre el presente trabajo, es que al ser un estudio transversal, resulta complicado generalizar inferencias causales entre las distintas variables, por lo que sería interesante plantear diseños longitudinales que pudieran dar mayor fiabilidad a las relaciones entre los diferentes constructos.

A nivel práctico, los resultados del presente trabajo ayudarán a docentes de la actividad física y comunidades educativas en el planteamiento de propuestas deportivas atrayentes, tanto en la dedicación escolar como extraescolar. Adaptar las actividades a las características personales y contextuales de sus adolescentes, atendiendo al fomento de las relaciones sociales y la búsqueda de nuevas sensaciones, que permitan conectar emocionalmente entre los iguales. Al mismo tiempo, la creatividad en las propuestas didácticas, en conexión con actividades que ellos pueden practicar en su barrio o localidad, favorecerán la adherencia, y permitirán a los adolescentes tomar responsabilidades y saborear la vivencia social, autorregulándose en la práctica y conociéndose mejor a sí mismos individualmente y en relación con los demás, influyendo en sus estados de bienestar a nivel físico, social y emocional, mejorando así la calidad de sus relaciones interpersonales.

\section{Referencias}

Álvarez, P., Carrasco, M. y Fustos, J. (2010). Relación de la empatía y género en la conducta prosocial y agresiva, en adolescentes de distintos tipos de establecimientos educacionales. Revista Iberoamericana de Psicología: Ciencia y Tecnología, 3(2), 27-36.

Alvariñas, M., Fernández, M. A. y López, C. (2009). Actividad física y percepciones sobre deporte y género. Revista de Investigación en Educación, 6, 113-122. 
AMM (2008). Revisión de la Declaración de Helsinki abierta a comentarios. 20 de junio de 2008 [en línea]. [Fecha de consulta: 24 de mayo de 2017]. Disponible en: http://www.wma.net/es/40news/20archives/2008/2008_08/index.html

Aróstegi, B., Goñi, A., Zubillaga, A. e Infante, G. (2012). El autoconcepto físico de jóvenes futbolistas de alto rendimiento. Cuadernos de Psicología del Deporte, 13(1), 9-14.

Caprara, G. V. y Cervone, D. (2000). Personality: Determinants, dynamics, and potentials. New York: Cambridge University Press; 2000.

Caprara, G. V., Steca, P., Zelli, A. y Capanna, C. (2005). A new scale for measuring adult's prosocialness. European Journal of Psychological Assessment, 21(2), 77-89. doi: $10.1027 / 1015-5759.21 .2 .77$

Caputi, M., Lecce, S., Pagnin, A. y Banerjee, R. (2012). Longitudinal effects of theory of mind on later peer relations: the role of prosocial behavior. Developmental Psychology, 48(1), 257. doi: 10.1037/a0025402

Carlo, G., Hausmann, A., Christiansen, S. y Randall, B. A. (2003). Sociocognitive and behavioral correlates of a measure of prosocial tendencies for adolescents. The Journal of Early Adolescence, 23(1), 107-134. doi: 10.1177/0272431602239132

Carlo, G., Mestre, M. V., McGinley, M. M., Tur-Porcar, A., Samper, P. y Opal, D. (2014). The protective role of prosocial behaviors on antisocial behaviors: The mediating effects of deviant peer affiliation. Journal of Adolescence,37(4), 359-366. doi: 10.1016/j.adolescence.2014.02.009

Carlo, G., Mestre, M. V., McGinley, M., Tur-Porcar, A., Samper, P. y Streit, C. (2013). The structure and correlates of a measure of prosocial moral reasoning in adolescents from Spain. European Journal of Developmental Psychology, 10(2), 174-189. doi: $10.1080 / 17405629.2012 .762909$

Cecchini, J. A., Fernández, J., González, C. y Arruza, J. A. (2008). Repercusiones del programa Delfos de educación en valores a través del deporte en jóvenes escolares. $R e-$ vista de Educación, 346, 167-186.

Darr, C. (2011). Measuring student engagement: The development of a scale for formative use. En S. Christenson, A. Reschly y C. Wylie (Eds.), Handbook of research on student engagement. New York: Springer.

De la Torre, M. J., García, M. C., de la Villa, M. y Casanova, P. F. (2008). Relaciones entre violencia escolar y autoconcepto multidimensional en adolescentes de Educación Secundaria Obligatoria. European Journal of Education and Psychology, 1(2), 57-70. doi: 10.30552/ejep.v1i2.9 
Dunn, E. W., Aknin, L. B. y Norton, M. I. (2008). Spending money on others promotes happiness. Science, 319(5870), 1687-1688. doi: 10.1126/science.1150952

Eisenberg, N., Fabes, R. A. y Spinrad, T. L. (2006). Prosocial development. En W. Damon y N. Eisenberg (Eds.), Handbook of child psychology, Vol. 3: Social, emotional and personality development (pp. 646-718). Nueva York: John Wiley \& Sons.

Esnaola, I. y Zulaika, L. M. (2009). Physical activity and physical self-concept in a simple of middle-aged Basque adults. Perceptual and Motor Skills, 108, 479-490. doi: 10.2466/pms.108.2.479-490

Fajardo, F., Maestre, M., Felipe, E., León, B. y Polo, M. I. (2017). Análisis del rendimiento académico de los alumnos de educación secundaria obligatoria según las variables familiares. Educación XX1, 20(1). doi: 10.5944/educXX1.14475

García, F. y Musitu, G. (2014). Autoconcepto Forma-5 (AF-5) (4ª Edición). Madrid: TEA Ediciones.

Gilman, R. y Anderman, E. M. (2006). The relationship between relative levels of motivation and intrapersonal, interpersonal, and academic functioning among older adolescents. Journal of School Psychology, 44(5), 375-391. doi: 10.1016/j.jsp.2006.03.004

González, J., Caprara, G., Garcés de los Fayos, E. y Zuffianó, A. (2014). Importance of the Development of Prosocial Behavior Through Physical Education in the Adolescents'Personality. Education in a Competitive and Globalizing World. Nova Publications. New York. USA

González, J. y Portolés, A. (2014). Actividad física extraescolar: relaciones con la motivación educativa, rendimiento académico y conductas asociadas a la salud. Revista Iberoamericana de Psicología del Ejercicio y el Deporte, 9(1), 51-65.

Goñi, E. e Infante, G. (2010). Actividad físico-deportiva, autoconcepto físico y satisfacción con la vida. European Journal of Education and Psychology, 3(2). doi: 10.30552/ejep.v3i2.52

Guevara, I. P., Cabrera, V. E. y Barrera, F. (2007). Contextual factors and moral emotions as predictors of psychological adjusment in the adolescence. Universitas Psychologica, 6(2), 269-283.

Inglés, C. J., Benavides, G., Redondo, J., García-Fernández, J. M., Ruiz-Esteban, C., Estévez, C. y Huescar, E. (2009). Conducta prosocial y rendimiento académico en estudiantes españoles de Educación Secundaria Obligatoria. Anales de Psicología. 25, 93-101.

Inglés, C. J., Delgado, B., García-Fernández, J. M., Ruiz-Esteban, C. y Díaz-Herrero, A. (2010). Sociometric types and social interaction styles in a simple of Spanish adoles- 
cents. Spanish Journal of Psychology, 13(2), 728-738. doi: $10.1017 / \mathrm{S} 1138741600002390$

Inglés, C. J., Martínez-González, A. E. y García-Fernández, J. M. (2013). Conducta prosocial y estrategias de aprendizaje en una muestra de estudiantes españoles de Educación Secundaria Obligatoria. European Journal of Education and Psychology, 6(1), 3353.

Janosz, M., Archambault, I., Morizot, J. y Pagani, L. S. (2008). School engagement trajectories and their differential predictive relations to dropout. Journal of Social Issues, 64(1), 21-40. doi: 10.1111/j.1540-4560.2008.00546.x

Jiménez, T. I., Estévez, E. y Murgui, S. (2014). Ambiente comunitario y actitud hacia la autoridad: relaciones con la calidad de las relaciones familiares y con la agresión hacia los iguales en adolescentes. Anales de Psicología, 30(3), 1086-1095. doi: 10.6018/analesps.30.3.160041

Kärtner, J. Keller, H. y Chaudhary, N. (2010). Cognitive and social influences on early prosocial behavior in two socio-cultural contexts. Developmental Psychology, 46(4), 905914. doi: $10.1037 / \mathrm{a} 0019718$

Kornbluh, M. y Neal, J. W. (2016). Examining the many dimensions of children's popularity Interactions between aggression, prosocial behaviors, and gender. Journal of Social and Personal Relationships, 33(1), 62-80. doi: 10.1177/0265407514562562

Kumru, A., Carlo, G., Mestre, M. y Samper, P. (2012). Prosocial moral reasoning and prosocial behavior among Turkish and Spanish adolescents. Social Behavior and Personality, 40(2), 205-214. doi: 10.2224/sbp.2012.40.2.205

Li, Y., Doyle Lynch, A., Kalvin, C., Liu, J. y Lerner, R. M. (2011). Peer relationships as a context for the development of school engagement during early adolescence. International Journal of Behavioral Development,35(4), 329-342. doi: $10.1177 / 0165025411402578$

Martínez, A. E., Inglés, C. J., Piqueras, J. A. y Ramos, V. (2010). Importancia de los amigos y los padres en la salud y el rendimiento escolar. Electronic Journal of Research in Education Psychology 20, 8(1), 111-138. doi: 10.25115/ejrep.v8i20.1376

Martínez-Martínez, F. D. y González-Hernández, J. (2017). Autoconcepto, práctica de actividad física y respuesta social en adolescentes. Relaciones con el rendimiento académico. Revista Iberoamericana de Educación, 73(1).

Mesurado, B., Richaud, M. C., Mestre, M. V., Samper-García, P., Tur-Porcar, A., Morales Mesa, S. A. y Viveros, E. F. (2014). Parental Expectations and Prosocial Behavior of 
Adolescents From Low-Income Backgrounds: A Cross-CulturalComparison Between Three Countries-Argentina, Colombia, and Spain. Journal of Cross-Cultural Psychology, 45(9), 1471-1488. doi: 10.1177/0022022114542284

Mikolajewski, A. J., Chavarria, J., Moltisanti, A., Hart, S. A. y Taylor, J. (2014). Examining the factor structure and etiology of prosociality. Psychological Assessment, 26(4), 1259. doi: $10.1037 / \mathrm{a} 0037132$

Oyarzún, G., Estrada, C., Pino, E. y Oyarzún, M. (2012). Habilidades sociales y rendimiento académico: una mirada desde el género. Acta Colombiana de Psicología, 15(2), 2128.

Padilla, M. T., García, S. y Suárez, M. (2010). Diferencias de género en el autoconcepto general y académico de estudiantes de $4^{\circ}$ de ESO. Revista de Educación, 352, 495-515.

Páez, M., Fachinnelli, C., Gutiérrez-Martínez, O. y Hernández, M. (2007). Un análisis de las relaciones entre distintas dimensiones del autoconcepto y el rendimiento académico en una muestra de adolescentes argentinos. Revista Mexicana de Psicología, 24(1), 77-84.

Plazas, E. A., Morón, M., Santiago, A., Sarmiento, H., Ariza, S. y Darío, C. (2010). Relaciones entre iguales, conducta prosocial y género desde la educación primaria hasta la universitaria en Colombia. Universitas Psychologica, 9, 357-369.

Revuelta, L. y Esnaola, I. (2011). Clima familiar deportivo y autoconcepto físico en la adolescencia. European Journal of Education and Psychology, 4(1). doi: 10.30552/ejep.v4i1.61

Richaud, M. C. y Mesurado, B. (2016). Las emociones positivas y la empatía como promotores de las conductas prosociales e inhibidores de las conductas agresivas. Acción Psicológica, 13(2), 31-42. doi: 10.5944/ap.13.2.17808

Rosenberg, M. (1979). Conceiving the self. New York: Basic Books.

Rowsell, H. C., Ciarrochi, J., Deane, F. P. y Heaven, P. C. (2016). Emotion identification skill and social support during adolescence: A three-year longitudinal study. Journal of Research on Adolescence, 26(1), 115-125. doi: 10.1111/jora.12175

Ruiz-Juan, F. y García, E. (2002). El abandono y la ausencia de práctica de actividad físicodeportiva de tiempo libre del alumnado de la universidad de Almería. Un estudio longitudinal. Revista Digital Lecturas: Educación Física y Deportes, 8, 47.

Samadzadeh, M., Abbasi, M. y Shahbazzadegan, B. (2011). Survey of relationship between psychological hardiness, thinking styles and social skills with high school student's 
academic progress in Arak city. Procedia-Social and Behavioral Sciences, 28, 286292. doi: 10.1016/j.sbspro.2011.11.055

Samper, P., Mestre, V. y Malonda, E. (2015). Evaluation of the Role of Intellectual and Socioemotional Variables in Solving Problems in Adolescence. Universitas Psychologica, 14(1), 287-298. doi: 10.11144/Javeriana.upsy13-5.ervi

Steger, M. F., Kashdan, T. B. y Oishi, S. (2008). Being good by doing good: Daily eudaimonic activity and well-being. Journal of Research in Personality, 42(1), 22-42. doi: 10.1016/j.jrp.2007.03.004

Stojiljković, S., Todorović, J., Đigić, G. y Dosković, Z. (2014). Teachers'self-concept and empathy. Procedia-Social and Behavioral Sciences, 116, 875-879. doi: 10.1016/j.sbspro.2014.01.313

Torstveit, L., Sütterlin, S. y Lugo, R. G. (2016). Empathy, Guilt Proneness, and Gender: Relative Contributions to Prosocial Behaviour. Europe's Journal of Psychology, 12(2), 260. doi: $10.5964 /$ ejop.v12i2.1097

Veenstra, R., Lindenberg, S., Oldehinkel, A. J., De Winter, A. F., Verhulst, F. C. y Ormel, J. (2008). Prosocial and antisocial behavior in preadolescence: Teachers' and parents' perceptions of the behavior of girls and boys. International Journal of Behavioral Development, 32(3), 243-251. doi: 10.1177/0165025408089274

Wang, M. T. y Fredricks, J. A. (2014). The reciprocal links between school engagement, youth problem behaviors, and school dropout during adolescence. Child Development, 85(2), 722-737. doi: 10.1111/cdev.12138

Wang, M. T. y Holcombe, R. (2010). Adolescents' perceptions of school environment, engagement, and academic achievement in middle school. American Educational Research Journal, 47(3), 633-662. doi: 10.3102/0002831209361209

Weinstein, N. y Ryan, R. M. (2010). When helping helps: An examination of motivational constructs underlying prosocial behavior and their influence on well-being for the helper and recipient. Journal of Personality and Social Psychology, 98(2), 222-224.

Wentzel, K. R. (2005). Peer relationships, motivation, and academic performance at school. En J. Elliot y C. S. Dweck (Eds.), Handbook of competence and motivation (pp. 279296). Nueva York: Guilford Press.

Zalagaz, M. L., Castro, R., Valdivia, P. y Cachón, J. (2017). Relación entre autoconcepto físico, ansiedad y personalidad manifestada en usuarios de gimnasios. Retos: Nuevas Tendencias en Educación Física, Deporte y Recreación (32), 53-57. 